\title{
Chromoendoscopy in combination with random biopsies does not improve detection of gastric cancer foci in CDH1 mutation positive patients
}

Authors

Institutions
Robert Hüneburg ${ }^{1,5,}$ * , Tim Marwitz ${ }^{1, ~ *}$, Peer van Heteren ${ }^{1,5}$, Tobias J. Weismüller ${ }^{1,5}$, Jonel Trebicka', Ronja Adam ${ }^{2,5}$, Stefan Aretz ${ }^{2,5}$, Alberto Perez Bouza ${ }^{3,5}$, Dimitrios Pantelis ${ }^{4,5}$, Jörg C. Kalff ${ }^{4,5}$, Jacob Nattermann ${ }^{1,5, *}$, Cristian P. Strassburg ${ }^{1,5}$, *

Institutions are listed at the end of article. submitted 6. January 2016 accepted after revision 13. June 2016

\section{Bibliography}

Dol http://dx.doi.org/ 10.1055/s-0042-112582 Published online: 31.8.2016 Endoscopy International Open 2016; 04: E1305-E1310

(c) Georg Thieme Verlag KG Stuttgart · New York E-ISSN 2196-9736

\section{Corresponding author} Robert Hüneburg, MD Department of Internal Medicine I

University of Bonn Sigmund-Freud Straße 25 D-53115 Bonn, Germany Phone: +49-228-287-16043 Fax: +49-228-287-19763 robert.hueneburg@ukb.unibonn.de
Background and study aims: Hereditary diffuse gastric cancer (HGGC), an autosomal dominant tumor-syndrome, accounts for $1 \%$ to $3 \%$ of gastric cancers worldwide. Presumably $30 \%$ to $40 \%$ of all patients fulfilling the clinical guidelines for HDGC are carriers of a pathogenic mutation in the $C D H 1$ gene. Patients often show multiple foci of signet ring cell carcinoma at early age and are advised to undergo prophylactic total gastrectomy (PTG). Our aim was to improve the endoscopic detection of HDGC by using an enhanced endoscopic protocol.

Patient and methods: Patients with a proven CDH1 germline mutation identified in our institute were prospectively included. Patients were advised to undergo PTG and offered a baseline endoscopic examination prior surgery. Examination was performed by using high-resolution white-light endoscopy and pan-gastric chromoendoscopy with indigo carmine as dye combined

\section{Introduction}

\section{$\nabla$}

Hereditary diffuse gastric cancer (HDGC) is an autosomal-dominant tumor-associated syndrome accounting for approximately $1 \%$ to $3 \%$ of gastric cancer (GC) [1]. In contrast to the intestinal-type GC (Laurén classification) the incidence of diffuse gastric cancer (DGC) is rising [2,3].

HDGC was described for the first time by Jones in 1964 in 1 Maori family [4]. In a subsequent study, researchers from New Zealand identified germline mutations of the $C D H 1$ gene, which encodes for the cell-to-cell adhesion protein E-Cadherin, as the molecular basis underlying familial clustering of gastric cancer in those families[5]. Carriers of the autosomal dominant inherited $C D H 1$ mutation have an estimated cumulative risk for GC at

\footnotetext{
* Drs. Hüneburg and Marwitz contributed equally and Dr. Strassburg shared senior authorship with Dr. Nattermann.
}

with targeted and multiple random biopsies assessed by an expert histopathologist. Postoperative histopathology was compared with results from endoscopic biopsies.

Results: Between September 2012 and November 20148 patients with a proven $C D H 1$ germline mutation were included. We conducted 44 targeted (6.3/patient) and 225 random (32.1/patient) biopsies in 7 patients. We detected 1 gastric cancer by random biopsy (14\%). All other examinations showed no signs of cancer.

Histopathology of gastrectomy specimen revealed multiple foci of gastric carcinoma in 6 patients (86\%) with a total number of 27 cancer foci. Conclusions: Examination with targeted and random biopsies combined with chromoendoscopy is not able to detect small foci of gastric cancer in CDH1 mutation carriers. Therefore PTG is advocated in these patients.

age 80 of $70 \%$ for men and $56 \%$ for women [6]. Others reported an even higher penetrance of over $80 \%$ in both men and women [7]. While clinically evident HDGC often occurs around the fourth decade, multiple foci of signet ring cell carcinoma (SRCC) can be found in young asymptomatic patients [8-11]. Additional cases found in Europe led to the creation of criteria for hereditary diffuse gastric cancer by the International Gastric Cancer Linkage Consortium (IGCLC) ( Table 1) [12-15]. In $25 \%$ to $50 \%$ of the families meeting HDGC criteria a pathogenic $C D H 1$ germline mutation can be identified [8]. Recent studies showed that HDGC is associated with other types of cancer especially lobular breast cancer (LBC) with an estimated lifetime risk of $42-60 \%$ for female carriers $[6,7,16]$.

At present HDGC patients with proven $C D H 1$ mutations are advised to undergo prophylactic total gastrectomy (PTG) before developing first symptoms of GC because of the very poor prognosis in 
Table 1 Clinical criteria for hereditary diffuse gastric cancer (HDGC) defined by the International Gastric Cancer Linkage Consortium guidelines (IDGLC) [15].

\begin{tabular}{|ll}
\hline 1 & $\begin{array}{l}\text { Two or more GC cases regardless of age, at least } 1 \text { confirmed DGC, } \\
\text { in first-degree and second-degree relatives }\end{array}$ \\
\hline 2 & $\begin{array}{l}\text { Isolated individual diagnosed with DGC at age }<40 \text { from a low } \\
\text { incidence population }\end{array}$ \\
\hline 3 & $\begin{array}{l}\text { Personal or family history of both DGC and LBC, with } 1 \text { case } \\
<50 \text { years at time of diagnosis }\end{array}$ \\
\hline
\end{tabular}

established disease [9,17-19]. Current recommendations suggest performing surgery between ages 16 and 30 or 5 years earlier than the age of the youngest relative at diagnosis of clinically apparent GC [20].

On the other hand, some $C D H 1$ mutation carriers may decline surgery because of concerns regarding long-term consequences as well as the risk of operation-associated complications [17]. Moreover, a significant number of these patients will never develop a $C D H 1$-associated gastric carcinoma, and thus, PTG is not needed in them.

Preoperative diagnosis of early DGC is hampered by the fact that the tumor cells begin infiltrating the mucosa while preserving a normal surface epithelium. Accordingly, rarely are any visible lesions spotted endoscopically. To overcome this obstacle, a variety of different endoscopic surveillance protocols have been studied in individuals with CDH1 mutations [10,21]. Some of these studies demonstrated that chromoendoscopy might increase diagnostic accuracy and thus suggested that endoscopy may have role in guiding the timing of total gastrectomy. However, even in these promising studies, endoscopic surveillance yielded falsenegative results in a significant proportion of patients $[10,21]$. Moreover, there are some concerns regarding the potential toxicity of methylene blue and congo - red, which have been used as staining agents in earlier chromoendoscopy studies [10,21]. This is in contrast to indigo carmine, a dye that has been widely used in upper [22] and lower gastrointestinal endoscopy [23,24]. Of note, indigo carmine has been shown to significantly increase detection rate of colorectal neoplasms [24]. To our knowledge no data exist whether use of this staining agent may have any role in surveillance of $\mathrm{CDH} 1$ mutation carriers and, thus, this was analyzed in the current study. To further increase the chance of detecting a malignant focus, chromoendoscopy with indigo carmine was combined with a minimum of 30 random biopsies per patient following a recommendation by the International Gastric Cancer Linkage Consortium (IGCLC) [15]. The diagnostic performance of this endoscopic surveillance protocol was studied in 8 carriers of a pathologic $C D H 1$ germline mutation that subsequently underwent prophylactic/therapeutic gastrectomy.

\section{Patients and methods}

$\nabla$

\section{Patients}

Between September 2012 and November 2014 patients with a proven $C D H 1$ germline mutation identified by routine molecular genetic diagnostics in our institute were included. All patients were managed by a multidisciplinary team of gastroenterologists, gastrointestinal oncologists, surgeons and geneticists in our center for hereditary tumor syndromes. Patients were referred for prophylactic total gastrectomy and all were offered baseline endoscopy of the upper digestive tract. Institutional review board approval was obtained (099/15).

The number of SRCC detected by targeted biopsies, random biopsies or a combination is reported as well as the number of SRCC in gastrectomy specimen. Follow up time is defined as the time span from first endoscopic examination to the most recent endoscopic examination.

Data are given as mean with standard deviation and proportion where appropriate.

\section{Endoscopy protocol}

Baseline endoscopy was performed following a standard protocol by a team of trained endoscopists with expertise in chromoendoscopy of the stomach. All patients were offered conscious sedation with propofol. Constant insufflation and exsufflation, changing of positions and application of a mucolytic agent ( $\mathrm{N}$-acetylcysteine), if needed, was done to ensure full view of all anatomical segments of the mucosa up to the descending part of the duodenum. The examination was conducted with a high-resolution endoscope (EG-590WR; Fujifilm Corporation, Tokyo, Japan). Pan-chromoendoscopy of the stomach is done by applying $0.4 \%$ indigo carmine dye (Indigo carmine Amino; Amino AG, Neuenhof Switzerland) via spray catheter (Olympus PW-5V1) to enhance contrast and to locate possible lesions. At least 25 minutes were spent to systematically examine the gastric mucosa. All suspicious lesions were described and biopsied. Furthermore a minimum of 30 random biopsies from all anatomical parts of the stomach (prepylorus, antrum, body, fundus and cardia) were taken.

\section{Pathology}

A designated gastrointestinal pathologist examined all biopsy and gastrectomy specimens. Gastrectomy specimens were opened along the greater curvature, pinned and stretched on cork plate and fixed with $4 \%$ buffered formalin overnight. Each stomach was entirely submitted for histological examination using $3 \mathrm{~mm}$ to $5 \mathrm{~mm}$-thick tissue sections that were routinely processed, paraffin-embedded, sectioned at $5 \mathrm{um}$ and stained with H\&E and also alcian-blue/PAS on serial sections. The topographical localisation of each sample was carefully annotated on a schematic map of the stomach.

Carcinomas were reported according to TNM classification of malignant tumors and protocolled along with every pathological finding.

\section{Results}

\section{$\nabla$}

\section{Patient characteristics and $\mathrm{CDH} 1$ status}

Eight patients from 3 different families with a history of either GC or LBC at a young age were included in our study. All the families fulfilled the extended criteria for HDGC [25]. In total 5 female and 3 male patients with a median age of 42.9 years (range 23 years to 61 years) at the time of study inclusion were analyzed. The clinical and genetic details of the patients are listed in Table 2. Genetic testing of the $C D H 1$ gene using leukocyte DNA revealed a missense mutation (c.1108G >A;p.Asp370Asn, exon 8) in family 1 , a nonsense mutation (c.2116C >T;p.Gln706*, exon 13) in family 2 , and a predicted splice site mutation (c.1137G $>A$, exon 8 ) in family 3 (GenBank RefSeq sequence NM_004360.3). The missense mutation c.1108G >A; p.Asp370Asn is located in a highly conserved region of the gene; 3 of 3 common in-silico tools (PolyPhen-2, MutationTaster, SIFT) predict this mutation to be prob- 


\begin{tabular}{|c|c|c|c|c|c|c|c|c|c|c|}
\hline Patient & Kindred & Sex & $\begin{array}{l}\text { Age } \\
\text { (years) }\end{array}$ & $\begin{array}{l}\text { CDH1 germline } \\
\text { mutation }\end{array}$ & $\begin{array}{l}\text { Positive } \\
\text { endoscopy }\end{array}$ & $\begin{array}{l}\text { Time interval } \\
\text { Endoscopy } \rightarrow \\
\text { surgery (days) }\end{array}$ & $\begin{array}{l}\text { Tumor loca- } \\
\text { lization and } \\
\text { number of } \\
\text { foci }\end{array}$ & $\begin{array}{l}\text { Total num- } \\
\text { ber of car- } \\
\text { cinomas }\end{array}$ & TNM & $\begin{array}{l}\text { Follow-up } \\
\text { (months) }\end{array}$ \\
\hline A & 1 & $\mathrm{~F}$ & 23 & \multirow{5}{*}{$\begin{array}{l}\text { c. } 1108 G>A \text {; } \\
\text { p.Asp } 370 \text { Asn } \\
\text { exon } 8\end{array}$} & No & 70 & $\begin{array}{l}\text { Cardia (1) } \\
\text { Body (3) }\end{array}$ & 4 & T1aNOMO & 7 \\
\hline B & 1 & M & 61 & & No & 71 & Body (5) & 5 & TisNOMO & 6 \\
\hline C & 1 & M & 27 & & No & 120 & & 0 & No tumor & \\
\hline $\begin{array}{l}\text { D } \\
\text { (excluded) }\end{array}$ & 1 & M & 39 & & No & 150 & & 0 & No tumor & \\
\hline $\mathrm{E}$ & 1 & $\mathrm{~F}$ & 43 & & No & 150 & Body (1) & 1 & T1aNOM0 & 24 \\
\hline $\mathrm{F}$ & 2 & $\mathrm{~F}$ & 52 & \multirow{2}{*}{$\begin{array}{l}\text { c. } 2116 C>T \text {; } \\
\text { p.Gln706* } \\
\text { exon } 13\end{array}$} & $\begin{array}{l}\text { Yes } \\
\text { (Carcinoma in } \\
\text { random biopsy) }\end{array}$ & 12 & $\begin{array}{l}\text { Body (2) } \\
\text { Fundus (4) }\end{array}$ & 6 & T1aNOM0 & 10 \\
\hline G & 2 & $\mathrm{~F}$ & 54 & & No & 13 & $\begin{array}{l}\text { Body (3) } \\
\text { Fundus (4) } \\
\text { Antrum (2) }\end{array}$ & 9 & T1aNOM0 & 8 \\
\hline $\mathrm{H}$ & 3 & $\mathrm{~F}$ & 44 & $\begin{array}{l}\text { c. } 1137 G>A \\
\text { exon } 8\end{array}$ & No & 1 & Body (2) & 2 & T1aNOM0 & 6 \\
\hline
\end{tabular}

ably pathogenic. The third mutation, c.1137G >A, is located within the splice donor site of exon 8.5 of 5 in-silico tools predict that it affects splicing (Human Splicing Finder, NNSPLICE, SpliceSiteFinder-like, MaxEntScan and GeneSplicer predicted the efficiency of the splice site reduced by $70 \%$ in average, range $13 \%$ to $100 \%$ ). This mutation was previously detected in a HDGC family and aberrant splicing of $C D H 1$ RNA was shown in one of the mutation carriers [26].

At initial presentation, none of the mutation carriers showed any signs of clinically evident stomach cancer such as abdominal pain, weight loss or dysphagia. However, 6 of the 8 patients (75\%) reported minor symptoms of dyspepsia.

\section{Endoscopic findings}

All patients underwent upper gastrointestinal endoscopy with an average examination time of 37.7 minutes (+/- 9). However, 1 patient (patient D) had to be excluded from further analysis due to violation of the endoscopy protocol, as only 8 random biopsies were taken.

Five of the remaining 7 patients (Patients A, B, G, H, E) had abnormal findings ( $\bullet$ Fig. 1), including flat polypoid lesions $<5 \mathrm{~mm}$ in the antrum (Patients A, G, E), erosions (Patient B), and erythema (Patients $\mathrm{E}, \mathrm{G}$ ) during examination of the gastric mucosa using chromoendoscopy. Pale lesions $(<3 \mathrm{~mm})$ were detected in $1 \mathrm{pa}-$ tient $(\mathrm{H})(\bullet$ Fig. 2$)$.

From these lesions 44 targeted biopsies (6.3/patient) were taken, revealing intestinal metaplasia of the stomach without any signs of dysplasia in histopathological examination.

In addition, 225 random biopsies (32.1/patient) were performed, which identified a single focus of SRCC $(0.3 \mathrm{~mm}$, pT1a) confined to the Lamina propria in patient $\mathrm{F}$ (figure 2).

Barrett's esophagus was diagnosed macroscopically and proven by histopathology in two individuals (Patients E, G), including one C8M1-Barett with high-grade dysplasia.

\section{Gastrectomy findings and correlation with endoscopy}

After an average interval of 62.4 days (+/- 53) following endoscopic examination all patients underwent PTG with D1 lymph node dissection in 3 and extended D2 lymph node dissection in 3 patients, respectively. In Patient $\mathrm{F}$, who was diagnosed with
SRCC in endoscopy, PTG with extended D2 lymph node dissection and omentectomy was performed.

The following extended, systematic macroscopic and microscopic examination of the gastrectomy specimen showed multifocal SRCC in 6 of the 7 patients (Patients A, B, E, F, G, H) ( Table 2 ). Carcinomas were located in body (16), fundus (8), cardia (1) and antrum (2) of the stomach ( Table 2). In total 27 foci of SRCC were detected.

No sign of lymphogenic tumor spread was observed in any of the lymph nodes that were removed. Giemsa staining of the gastrectomy specimen displayed no infection of Helicobacter pylori.

During follow up, all 6 patients with proven cancer received control endoscopy of the upper digestive tract a median of 8 months (range 6 months to 10 months) after surgery, which did not reveal any suspicious lesion. Biopsies taken proved to be free of cancer.

\section{Discussion}

In carriers of a pathogenic $C D H 1$ germline mutation, current guidelines recommend PTG for mutation carriers even if endoscopic biopsies have been negative [20]. However, the decisionmaking process is complex in these patients and some of $C D H 1$ mutation (+) patients might refuse PTG as they are afraid of a negative impact on quality of life and/or the risk of operationassociated complications $[17,27]$. Moreover, with a penetrance of up to $70 \%$, a relevant proportion of mutation carriers will never develop a CDH1-associated gastric carcinoma and thus PTG is not warranted in these patients. Therefore, there is a strong need to improve endoscopic surveillance in CDH1-mutation carriers in order to allow reliable detection of early gastric cancer, thereby enabling prompt decision making.

Here, we analyzed the diagnostic performance of chromoendoscopy using indigo carmine dye in combination with random biopsies for the detection of SRCC in 7 CDH1 germline mutation carriers from 3 different families. In random biopsies SRCC was detected in one female patient (14\%), whereas chromoendoscopy-guided targeted biopsies failed to detect any cancer. In contrast, final histopathologic analysis of the gastrectomy specimen 


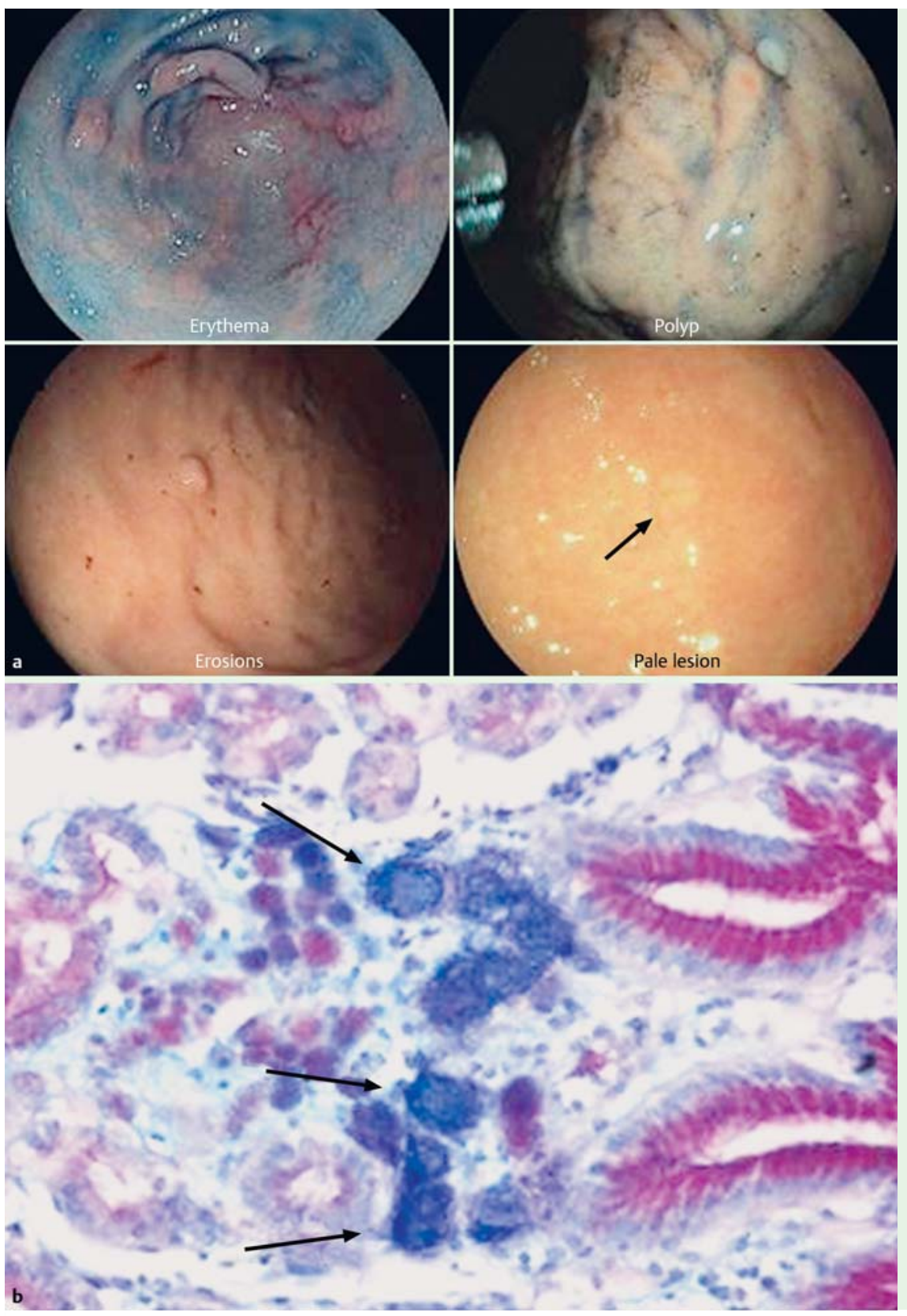

Fig. 1 a Examples of endoscopic lesions targeted by biopsy but negative for malignant cells at histopathological analysis. b Histopathologic diagnosis of SRCC in a random biopsy stained with alcian-blue and PAS. Signet ring cells are depicted by the strongly blue reaction due to the acidic mucine in the cells. Tumor cells are located in the lamina propria of the mucosa

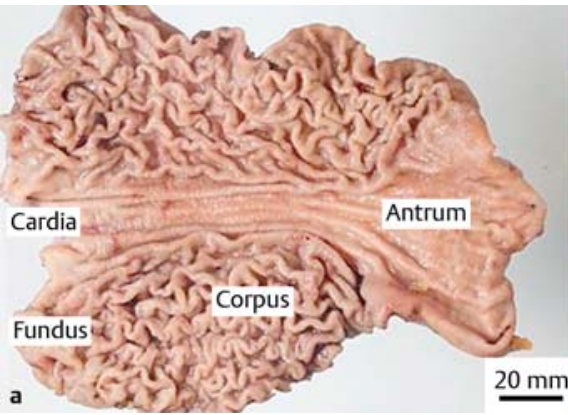

$\underline{20 \mathrm{~mm}}$

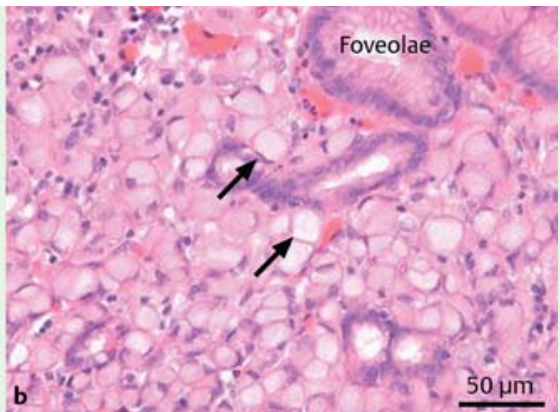

Fig. 2 a Gastrectomy specimen opened along the greater curvature with no macroscopic sign of cancer. $\mathbf{b}$ Histologic image of the mucosa stained with H\&E showing a focus of SRCC (arrow) detected a total of 27 SRCC foci in 6 of the 7 patients (86\%); indicating that chromoendoscopy in combination with random biopsies does not allow a reliable detection of malignant foci in $\mathrm{CDH} 1$ mutation carriers.

This is in contrast to earlier studies which suggested that chromoendoscopy-guided targeted biopsies or careful white-light examination with targeted and random biopsies can identify early lesions, thereby helping to inform decision making with regard to gastrectomy $[10,21]$.

For instance, Shaw et al. demonstrated that chromoendoscopy with methylene blue and congo-red as staining agents facilitated the detection of early gastric carcinoma foci not visible by white light endoscopy [10]. However, in this study the pale lesions identified by chromoendoscopy appeared to correspond to the larger 
malignant foci of $4 \mathrm{~mm}$ to $10 \mathrm{~mm}$, whereas diagnostic performance for smaller lesions was low [10]. In our cohort, all of the cancer foci found after gastrectomy were smaller than $4 \mathrm{~mm}$. Thus, chromoendoscopy seems to be insufficient for sensitive detection of small SRCC foci, irrespective of the dye used for staining. Moreover, there is wide variation in the number of these foci both within families and between HDGC kindreds, with average values ranging from $<20$ to $>100$ foci $[8,16,18,19,28]$. Indeed, the total number of SRCC foci per patient was much higher in the study by Shaw and co-workers than in our cohort which may explain the higher detection rate in the study by Shaw et al. (157 foci/patient vs. 4.5 foci/patient).

In a more recent study, Lim et al. evaluated the diagnostic performance of high-resolution white-light endoscopy with autofluorescence (AFI) and narrow-band imaging (NBI). In this work, the authors stated that very few targeted biopsies corresponded to SRCC (9.6\%). Accordingly, only 5 of 16 patients with SRCC foci were identified by this approach. This further emphasizes the difficulties in sensitive detection of malignant foci by targeted biopsies.

Taking into account this problem the IGCLC established the Cambridge protocol for endoscopic examination [25], stating that a minimum of 30 random biopsies per patient should be taken. In line with this recommendations a total of 226 (31.1/patient) random biopsies were performed in our study. However, by this approach false-negative results were obtained in 6 out of 7 studied patients with a single focus of SRCC identified in 1 out of 226 random biopsies $(0.4 \%)$. In the study of Lim et al. who performed an average of 24 random biopsies/ patient SRCC foci were detected with a sensitivity of about $80 \%$ (13/16 patients) [21], which is markedly higher than in our study (14.3\%). This discrepancy most likely reflects the lower number of cancer foci found in gastrectomy specimen of our patients compared to that analyzed by Lim and co-workers (4.5/patient vs. 62/patient). Of note, even in the context of this rather high number of malignant foci 3 out of 16 patients positive for SRCC were missed by random biopsies in the Lim et al. study. This is in line with a report by Fujita et al. who calculated the theoretical number of biopsies necessary to capture at least 1 cancer focus to be 1768 (range 50-5832) to assure a $90 \%$ detection rate [29]. For obvious reasons this is clinically unsustainable.

Several series have studied the predilection of cancer foci in gastrectomy specimens $[8,16,18,30]$. In our study, the majority of SRCC foci were found in body $(16 / 27,59 \%)$ and fundus $(8 / 27$, $30 \%)$, which is in line with data reported by Lim et al. and Fujita and co-workers. However, localization of DGC varied between different studies and thus there still is no agreement as to whether the proximal $[16,30]$ or the transitional zone $[8,18]$ should be preferentially sampled.

HDGC is a rare disease and, thus, it would not have been feasible to include a control cohort, which is a major limitation of our analysis. However, the observational character of our study reflects a real life scenario for each of the patients and highlights the current problems in correct diagnostics and subsequent decision-making in CDH1 mutation carriers.

In our cohort all patients underwent gastrectomy. In 6 of 8 patients, histopathologic analysis of the gastrectomy specimen revealed multifocal SRCC, including 5 patients with negative results on endoscopy. All patients displayed early stages of SRCC (Tis or T1a) and thus did not need any adjuvant chemotherapy, which further supports the idea of prophylactic surgery in HDGC patients.
However, at what stage to recommend PTG remains debatable. Risk of advanced HDGC is considered to be less than $1 \%$ at age 20 years. Accordingly, some authors suggest that gastrectomy should be applied during the second decade of life because risk of metastases in patients younger than age 20 years is low [16, 31]. However, that is controversial as other studies have described patients who died of gastric cancer in their early $20 \mathrm{~s}$ [9]. In this context, sensitive identification of foci that are beginning to progress or which display specific gene expression signatures [32-37] suggesting a high likelihood of progression would be of great diagnostic benefit. Future studies are needed to clarify whether novel endoscopic methods such as confocal endomicroscopy [38] in combination with molecular analysis of targeted biopsies [39] may be helpful to achieve this goal.

\section{Conclusions}

Taken together, our findings indicate that chromoendoscopy in combination with random biopsies does not enable reliable and sensitive detection of SRCC foci in patients with known $C D H 1$ mutation and emphasize the need for novel endoscopic techniques enabling more refined strategies for mucosal evaluation and biopsy targeting in these patients.

\section{Competing interests: None}

\section{Institutions}

${ }^{1}$ Department of Internal Medicine I, University Hospital Bonn, Bonn, Germany

2 Institute of Human Genetics, University Hospital Bonn, Bonn, Germany

${ }^{3}$ Institute of Pathology, University Hospital Bonn, Bonn, Germany

${ }^{4}$ Department of Surgery, University Hospital Bonn, Bonn, Germany

${ }^{5}$ Center for Hereditary Tumor Syndromes, University Hospital Bonn, Bonn, Germany

\section{Acknowledgements}

This work was supported by BONFOR (2014-11B-03), the German Research Foundation (DFG SFB/TRR 57), and the H. W. and J. Hector Foundation [grant number M69].

\section{References}

1 Palli D, Galli M, Caporaso NE et al. Family history and risk of stomach cancer in Italy. Cancer Epidemiology Biomarkers \& Prevention 1994; 3: $15-18$

2 Henson DE, Dittus $C$, Younes $M$ et al. Differential Trends in the Intestinal and Diffuse Types of Gastric Carcinoma in the United States, 19732000: Increase in the Signet Ring Cell Type. Archives of Pathology \& Laboratory Medicine 2004; 128: 765 - 770

3 Howson CP, Hiyama T, Wynder EL. The decline in gastric cancer: epidemiology of an unplanned triumph. Epidemiologic reviews 1986; 8: 1 27

4 Jones EG. Familial Gastric Cancer. The New Zealand medical journal 1964; 63: $287-296$

5 Guilford P, Hopkins J, Harraway J et al. E-cadherin germline mutations in familial gastric cancer. Nature 1998; 392: 402-405

6 Hansford S, Kaurah P, Li-Chang H et al. Hereditary diffuse gastric cancer syndrome: Cdh1 mutations and beyond. JAMA Oncology 2015; 1: 23 32

7 Molinaro V, Pensotti V, Marabelli $M$ et al. Complementary molecular approaches reveal heterogeneous $\mathrm{CDH} 1$ germline defects in Italian patients with hereditary diffuse gastric cancer (HDGC) syndrome. Genes, chromosomes \& cancer 2014; 53: 432 - 445

8 Oliveira C, Senz J, Kaurah P et al. Germline CDH1 deletions in hereditary diffuse gastric cancer families. Hum Mol Genet 2009; 18: 1545 - 1555

9 Chen $Y$, Kingham $K$, Ford JM et al. A prospective study of total gastrectomy for CDH1-positive hereditary diffuse gastric cancer. Annals of surgical oncology 2011; 18: $2594-2598$ 
10 Shaw D, Blair V, Framp A et al. Chromoendoscopic surveillance in hereditary diffuse gastric cancer: an alternative to prophylactic gastrectomy? Gut 2005; 54: $461-468$

11 Mayrbaeurl B, Keller G, Schauer W et al. Germline mutation of the Ecadherin gene in three sibling cases with advanced gastric cancer: clinical consequences for the other family members. European journal of gastroenterology \& hepatology 2010; 22: 306 - 310

12 Keller G, Vogelsang $H$, Becker I et al. Diffuse type gastric and lobular breast carcinoma in a familial gastric cancer patient with an E-cadherin germline mutation. The American journal of pathology 1999; 155: $337-342$

13 Gayther SA, Gorringe KL, Ramus SJ et al. Identification of germ-line Ecadherin mutations in gastric cancer families of European origin. Cancer research 1998; 58: 4086-4089

14 Guilford PJ, Hopkins JB, Grady WM et al. E-cadherin germline mutations define an inherited cancer syndrome dominated by diffuse gastric cancer. Human mutation 1999; 14: 249-255

15 van der Post RS, Vogelaar IP, Carneiro F et al. Hereditary diffuse gastric cancer: updated clinical guidelines with an emphasis on germline CDH1 mutation carriers. J Med Genet 2015; 52: 361 - 374

16 Pharoah PD, Guilford P, Caldas $C$ et al. Incidence of gastric cancer and breast cancer in $\mathrm{CDH} 1$ (E-cadherin) mutation carriers from hereditary diffuse gastric cancer families. Gastroenterology 2001; 121: 1348 1353

17 Lynch HT, Kaurah P, Wirtzfeld D et al. Hereditary diffuse gastric cancer: diagnosis, genetic counseling, and prophylactic total gastrectomy. Cancer 2008; 112: 2655 - 2663

18 Pandalai PK, Lauwers GY, Chung DC et al. Prophylactic total gastrectomy for individuals with germline CDH1 mutation. Surgery 2011; 149: 347-355

19 Hebbard PC, Macmillan A, Huntsman D et al. Prophylactic total gastrectomy (PTG) for hereditary diffuse gastric cancer (HDGC): the Newfoundland experience with 23 patients. Annals of surgical oncology 2009; 16: 1890-1895

20 Seevaratnam R, Coburn N, Cardoso $R$ et al. A systematic review of the indications for genetic testing and prophylactic gastrectomy among patients with hereditary diffuse gastric cancer. Gastric cancer : official journal of the International Gastric Cancer Association and the Japanese Gastric Cancer Association 2012; 15: 01153 - 163

21 Lim YC, di Pietro M, O'Donovan M et al. Prospective cohort study assessing outcomes of patients from families fulfilling criteria for hereditary diffuse gastric cancer undergoing endoscopic surveillance. Gastrointestinal endoscopy 2014; 80: $78-87$

22 Dekker E, Boparai KS, Poley JW et al. High resolution endoscopy and the additional value of chromoendoscopy in the evaluation of duodenal adenomatosis in patients with familial adenomatous polyposis. Endoscopy 2009; 41: $666-669$

23 Huneburg $R$, Lammert F, Rabe C et al. Chromocolonoscopy detects more adenomas than white light colonoscopy or narrow band imaging colonoscopy in hereditary nonpolyposis colorectal cancer screening. Endoscopy 2009; 41: 316-322
24 Kahi CJ, Anderson JC, Waxman I et al. High-definition chromocolonoscopy vs.high-definition white light colonoscopy for average-risk colorectal cancer screening. The American journal of gastroenterology 2010; 105: $1301-1307$

25 Fitzgerald RC, Hardwick $R$, Huntsman $D$ et al. Hereditary diffuse gastric cancer: updated consensus guidelines for clinical management and directions for future research. J Med Genet 2010; 47: 436-444

26 Frebourg T, Oliveira C, Hochain P et al. Cleft lip/palate and CDH1/E-cadherin mutations in families with hereditary diffuse gastric cancer. Journal of Medical Genetics 2006; 43: 138-142

27 Choi JH, Kim ES, Lee YJ et al. Comparison of quality of life and worry of cancer recurrence between endoscopic and surgical treatment for early gastric cancer. Gastrointestinal endoscopy 2015; 82: 299-307

28 Rogers WM, Dobo E, Norton JA et al. Risk-reducing total gastrectomy for germline mutations in E-cadherin (CDH1): pathologic findings with clinical implications. Am J Surg Pathol 2008; 32: 799-809

29 Fujita H, Lennerz JK, Chung DC et al. Endoscopic surveillance of patients with hereditary diffuse gastric cancer: biopsy recommendations after topographic distribution of cancer foci in a series of $10 \mathrm{CDH} 1$-mutated gastrectomies. Am J Surg Pathol 2012; 36: 1709-1717

30 Kaurah P, MacMillan A, Boyd N et al. Founder and recurrent cdh1 mutations in families with hereditary diffuse gastric cancer. JAMA 2007; 297: $2360-2372$

31 Newman EA, Mulholland MW. Prophylactic Gastrectomy for Hereditary Diffuse Gastric Cancer Syndrome. Journal of the American College of Surgeons 2006; 202: 612-617

32 Brettingham-Moore $K H$, Duong $C P$, Heriot AG et al. Using Gene Expression Profiling to Predict Response and Prognosis in Gastrointestinal Cancers - The Promise and the Perils. Annals of surgical oncology 2011; 18: $1484-1491$

33 Chibon F. Cancer gene expression signatures - The rise and fall? European journal of cancer 2013; 49: 2000-2009

34 Boussioutas A, Li H, Liu J et al. Distinctive Patterns of Gene Expression in Premalignant Gastric Mucosa and Gastric Cancer. Cancer Research 2003: 63: 2569-2577

35 Vecchi M, Nuciforo P, Romagnoli S et al. Gene expression analysis of early and advanced gastric cancers. Oncogene 2007; 26: 4284-4294

$36 \mathrm{Nam} \mathrm{S}$, Lee J, Goh S-H et al. Differential gene expression pattern in early gastric cancer by an integrative systematic approach. International Journal of Oncology 2012; 41: 1675-1682

$37 \mathrm{Kim} \mathrm{H}$, Eun JW, Lee H et al. Gene expression changes in patient-matched gastric normal mucosa, adenomas, and carcinomas. Experimental and Molecular Pathology 2011; 90: 201 - 209

38 Lee SK. Usefulness and Future Prospects of Confocal Laser Endomicroscopy for Gastric Premalignant and Malignant Lesions. Clin Endosc 2015; 48: $511-515$

39 Lin $X$, Zhao $Y$, Song $W$-m et al. Molecular classification and prediction in gastric cancer. Computational and Structural Biotechnology Journal 2015; $13: 448-458$ 